\title{
A Spectrum Sensing Method Based on Signal Feature and Clustering Algorithm in Cognitive Wireless Multimedia Sensor Networks
}

\author{
Yongwei Zhang, ${ }^{1}$ Pin Wan, ${ }^{1}$ Shunchao Zhang, ${ }^{1}$ Yonghua Wang, ${ }^{1,2}$ and Nan $\mathrm{Li}^{1}$ \\ ${ }^{1}$ School of Automation, Guangdong University of Technology, Guangzhou 510006, China \\ ${ }^{2}$ Key Laboratory of Machine Intelligence and Advanced Computing (Sun Yat-Sen University), Ministry of Education, \\ Guangzhou 510006, China
}

Correspondence should be addressed to Yonghua Wang; sjzwyh@163.com

Received 15 August 2017; Revised 26 September 2017; Accepted 4 October 2017; Published 26 October 2017

Academic Editor: Zhaoqing Pan

Copyright (C) 2017 Yongwei Zhang et al. This is an open access article distributed under the Creative Commons Attribution License, which permits unrestricted use, distribution, and reproduction in any medium, provided the original work is properly cited.

In order to solve the problem of difficulty in determining the threshold in spectrum sensing technologies based on the random matrix theory, a spectrum sensing method based on clustering algorithm and signal feature is proposed for Cognitive Wireless Multimedia Sensor Networks. Firstly, the wireless communication signal features are obtained according to the sampling signal covariance matrix. Then, the clustering algorithm is used to classify and test the signal features. Different signal features and clustering algorithms are compared in this paper. The experimental results show that the proposed method has better sensing performance.

\section{Introduction}

The Cognitive Wireless Multimedia Sensor Networks (CWMSN) have the function of real-time monitoring, collecting multimedia information, and a wide application background. Compared to the traditional wireless sensing networks, the CWMSN require greater bandwidth. So the CWMSN must have the ability to perceive the surrounding radio environment within a certain frequency range, which is called spectrum sensing ability. Spectrum sensing can determine which portions of the spectrum are available and detect the presence of licensed users when a user operates in a licensed band. With the wide application of wireless communication equipment, limited available spectrum resources are increasingly lacking. Cognitive radio (CR) can be used to solve the conflicts between spectrum scarcity and spectrum underutilization. It can improve the spectrum utilization by allowing secondary users to borrow unused radio spectrum from primary users or to share the spectrum with the primary users. Spectrum sensing technology is the key technology of cognitive radio. The main purpose is to detect the spectrum cavity and improve the efficiency of the spectrum [1-4]. Conventional spectrum sensing methods include energy detection, matched filter detection, and cyclostationary feature detection. Because the energy detection algorithm has low computational complexity and does not require a priori information about the primary users, it is the most common detection method for spectrum sensing. However, the wireless communication signal in the actual environment will include noise; this will seriously affect the perceived performance of the cognitive radio networks for the spectrum and reduce the probability of spectrum detection. In recent years, the method of random matrix theory has been applied to the spectrum sensing $[5,6]$, which uses the eigenvalue of the signal covariance matrix as the statistic and then derives the corresponding threshold to judge. For example, the ratio of the maximum or average eigenvalue to the minimum eigenvalue can be used to detect the presence of the signal [2]. However, the spectrum sensing based on random matrix theory has an inaccurate estimation problem, which directly affects the detection performance of the spectrum. Spectrum sensing can also be considered a problem of two categories (PU presence and PU absence). Therefore, the spectrum 
sensing method based on machine learning has gradually become the hotspot of people's research. A spectrum sensing method based on $k$-means clustering is proposed in [7]. This method takes the energy of the signal as a feature and then divides the features into channel available class and channel unavailable class by $K$-means clustering algorithm. The spectrum sensing method combined with the maximum minimum eigenvalue of support vector machine and signal covariance matrix is introduced in $[8,9]$. To overcome the shortcoming of spectrum sensing method based on random matrix, in this paper, we propose a spectrum sensing method based on signal features and clustering algorithm. The clustering algorithm uses the feature of the wireless communication signal to determine the PU status. In the experimental section, we use three different eigenvalues of the covariance matrix of the received signal, that is, the MME (the difference between the maximum eigenvalue and the minimum eigenvalue), MSE (the difference between the maximum eigenvalue and the mean eigenvalue), and RMET (the ratio of the maximum eigenvalue to the matrix trace). The spectrum sensing performance under different clustering algorithms ( $K$-means and $K$-medoids) is also analyzed. The simulation results show that the proposed method has good sensing performance.

\section{System Model}

2.1. System Structure. In CWMSN, many factors in practice such as multipath fading, shadowing, and the receiver uncertainty problem may significantly compromise the detection performance in spectrum sensing. In Figure 1, we can see that, due to multiple attenuated copies of the PU signal and the blocking of house, SU4 experiences multipath and shadow fading such that the PU's signal may not be correctly detected. However, the SU users can cooperate and share the sensing results with other users; the combined cooperative decision derived from spatially collected observations can overcome the deficiency of individual observations at each CR user. So cooperative spectrum sensing is an attractive and effective approach to combat multipath fading and shadowing and mitigate the receiver uncertainty problem [10].

2.2. Feature Extraction. It is assumed that there are $K$ SUs in the cognitive radio network and the number of sampling points per SU is $N$. We assume that $H_{0}$ indicates that the primary user signal does not exist and $H_{1}$ indicates that the primary user signal exists. Thus the model of sensing signal under both assumptions can be represented by the following equation:

$$
X_{i}(n)= \begin{cases}w_{i}(n), & H_{0} i=1,2, \ldots, K, \\ s_{i}(n)+w_{i}(n), & H_{1} i=1,2, \ldots, K,\end{cases}
$$

where $s_{i}(n)$ denotes the primary user (PU) signal and $w_{i}(n)$ denotes the Gaussian white noise signal, where the mean is 0 and the variance is $\sigma^{2}$. Let $S=1$ and $S=0$, respectively, indicate the state of PU. Channel availability $A$ can be defined as

$$
A= \begin{cases}0, & S=1, \\ 1, & S=0,\end{cases}
$$

where $A=0$ means that the channel cannot be used and $A=$ 1 means that the channel can be used.

Assuming that $X=\left[x_{i}(1), x_{i}(2), \ldots, x_{i}(N)\right]$ represents the sampling vector of the $i$ th SU user, the sensing matrix over a period of time can be expressed as

$$
X=\left[\begin{array}{cccc}
x_{1}(1) & x_{1}(2) & \cdots & x_{1}(N) \\
x_{2}(1) & x_{2}(2) & \cdots & x_{2}(N) \\
\vdots & \vdots & \ddots & \vdots \\
x_{k}(1) & x_{k}(2) & \cdots & x_{k}(N)
\end{array}\right]
$$

The covariance matrix of the signal can be expressed as $R(N)=(1 / N) X X^{T}$. In the traditional spectrum sensing technologies based on the random matrix theory, we usually select MME, MSE, and RMET as signal features. After selecting the feature, the corresponding threshold of the derivation is judged. These methods have the problem of threshold accuracy. In order to solve this problem, we introduce the clustering algorithm in machine learning, and we turn the dimension of the feature from one dimension to two dimensions.

The signal covariance matrix is expressed as $R(N)=$ $(1 / N) X X^{T}$. In order to obtain the two-dimensional features, we introduce the method of signal decomposition which is the $I$ and $Q$ decomposition before obtaining the sampling matrix [11], and the expression is as follows:

$$
\begin{gathered}
X_{i}^{I}=X_{i} * \sin \left(\frac{2 \pi f_{c} n}{f_{s}}\right) \quad n=1,2, \ldots, N, \\
X_{i}^{\mathrm{Q}}=X_{i} * \cos \left(\frac{2 \pi f_{c} n}{f_{s}}\right) \quad n=1,2, \ldots, N .
\end{gathered}
$$

After $I$ and $Q$ decomposition, we can get two sampling matrices:

$$
\begin{gathered}
X^{I}=\left[\begin{array}{cccc}
X_{1}^{I}(1) & X_{1}^{I}(2) & \cdots & X_{1}^{I}(N) \\
X_{2}^{I}(1) & X_{2}^{I}(2) & \cdots & X_{2}^{I}(N) \\
\vdots & \vdots & \ddots & \vdots \\
X_{m}^{I}(1) & X_{m}^{I}(2) & \cdots & X_{m}^{I}(N)
\end{array}\right], \\
X^{\mathrm{Q}}=\left[\begin{array}{cccc}
X_{1}^{\mathrm{Q}}(1) & X_{1}^{\mathrm{Q}}(2) & \cdots & X_{1}^{\mathrm{Q}}(N) \\
X_{2}^{\mathrm{Q}}(1) & X_{2}^{\mathrm{Q}}(2) & \cdots & X_{2}^{\mathrm{Q}}(N) \\
\vdots & \vdots & \ddots & \vdots \\
X_{m}^{\mathrm{Q}}(1) & X_{m}^{\mathrm{Q}}(2) & \cdots & X_{m}^{\mathrm{Q}}(N)
\end{array}\right] .
\end{gathered}
$$

So we can get the covariance matrices $R^{I}(N)=(1 /$ $N) X^{I}\left(X^{I}\right)^{T}$ and $R^{\mathrm{Q}}(N)=(1 / N) X^{\mathrm{Q}}\left(X^{\mathrm{Q}}\right)^{T}$. Then we calculate 


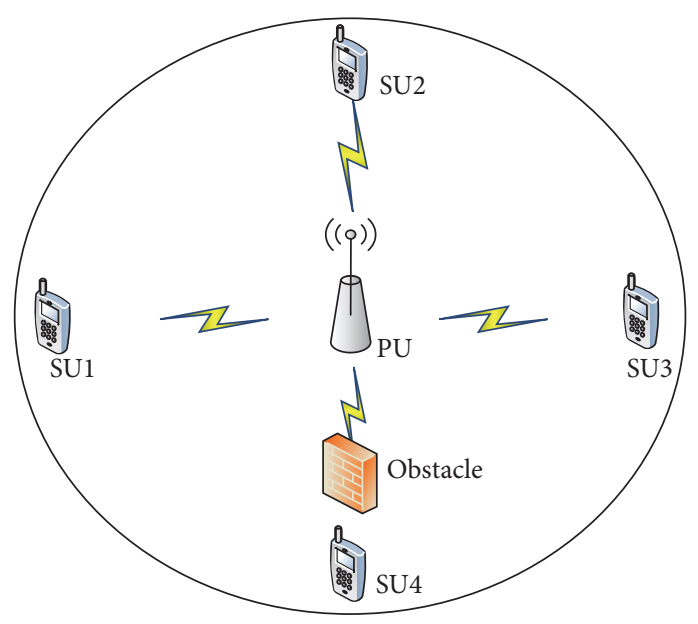

FIgURE 1: System structure.

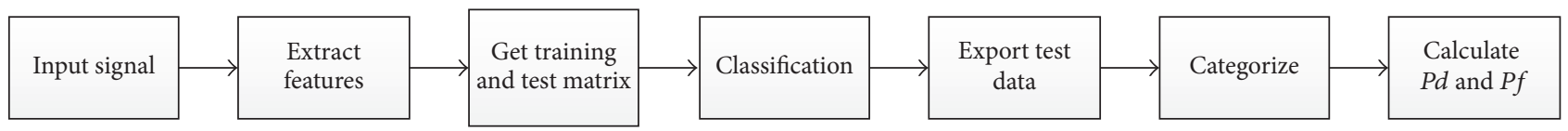

FIgURE 2: The system model.

the corresponding eigenvalues $T^{1}$ and $T^{2}$ for each covariance matrix and finally let $T=\left[T^{1}, T^{2}\right]$ represent the signal feature of each group. The false alarm probability and detection probability of the system can be defined as

$$
\begin{aligned}
& P f=p[A=1 \mid A=0], \\
& P d=p[A=0 \mid A=1] .
\end{aligned}
$$

\subsection{Spectrum Sensing Method Based on Feature and Clustering Algorithm}

2.3.1. Spectrum Sensing Scheme Based on Clustering Algorithm. In this paper, we propose novel CSS method based on classification algorithm. According to the type of learning method adopted, a classification algorithm can be categorized as unsupervised learning or supervised learning. We select two clustering algorithms to classify the feature, which are $K$-means clustering algorithm and $K$-medoids clustering algorithm. The two algorithms are similar, and the difference lies in the selection of centroids. The $K$-means algorithm uses the mean of all data points in the current cluster as centroids; however, the $k$-medoids algorithm selects the smallest points of distance from one to all points as centroids. The other steps are the same as the $K$-means algorithm.

The unsupervised $K$-means and $K$-medoids clustering algorithm partitions a set of the training feature vectors (i.e., $\left.\bar{T}=\left\{T_{1}, T_{2}, \ldots, T_{N}\right\}\right)$ into $k$ clusters. Considering the actual situation of spectrum sensing, the training matrix is divided into two classes. One class represents the noise, and the other represents the signal and the noise. After the training process is completed, the test data is put into the classifier, which can be judged by the following formula:

$$
\frac{\left\|\widehat{T}-\varphi_{1}\right\|}{\min _{K=2,3, \ldots, k}\left\|\widehat{T}-\varphi_{k}\right\|} \geq \varepsilon .
$$

If the data of the test matrix satisfies formula (7), it is judged that $A=0$; if it is not satisfied, then $A=1$.

The whole training and testing flow chart is shown in Figure 2.

\section{Experiments}

In order to show the effect of the proposed spectrum sensing algorithm, MME, MSE, and RMET are selected as the feature vectors in the simulation experiment. The experimental results are as follows.

3.1. The Classification Effect of Clustering Algorithm. We use AM signal in this experiment. The carrier frequency is $702 \mathrm{KHz}$ and the sampling rate is $4 \mathrm{MHz}$. In order to simulate the real environment, we did a simulation experiment under the Rayleigh channel. To ensure the accuracy of the experiment, we extracted 1000 signal features; 500 of them are training sets and the other 500 are test sets.

(1) We experimented with MME plus $k$-means clustering algorithm in the environment of SNR $=-10$ and SNR = -15 , respectively. Figures 3 and 4 show that the training data are divided into two clusters. One cluster represents the channel available class and the other represents the channel unavailable class. We can see that the classification result is better in high SNR environment. 


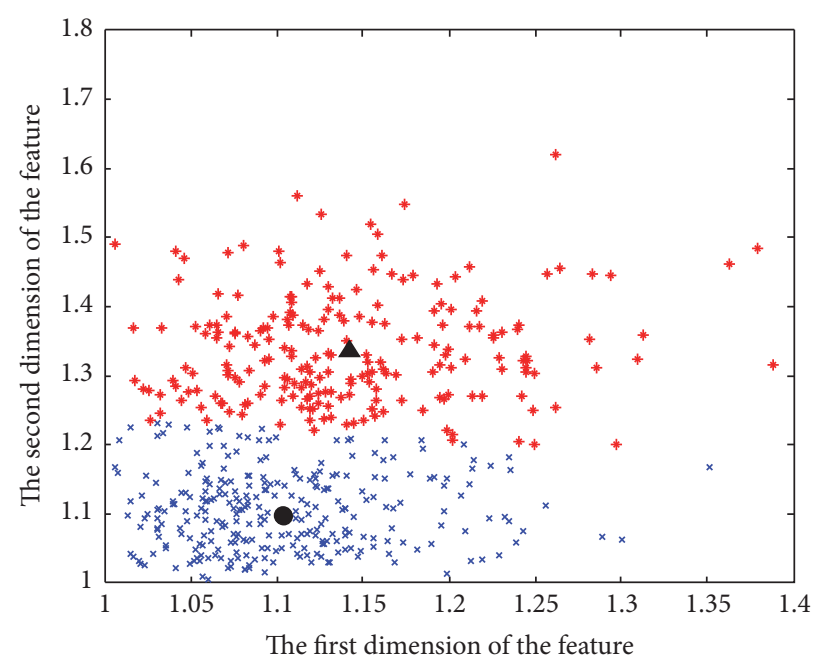

FIgURE 3: The clustering map under $K$-means algorithm.

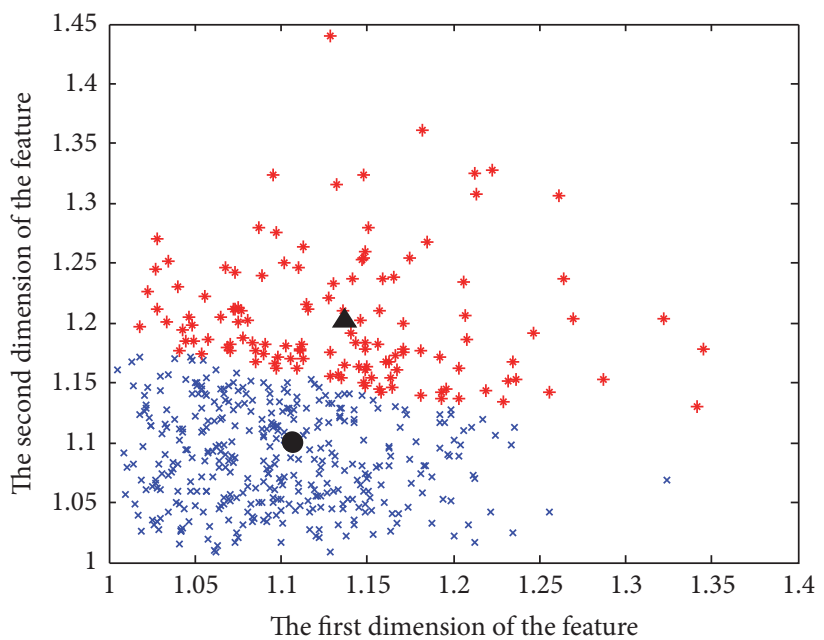

FIgURE 4: The clustering map under $K$-means algorithm.

(2) We experimented with MME plus $k$-medoids clustering algorithm in the environment of SNR $=-10$ and SNR = -15 , respectively. From Figures 5 and 6 , we can also find that the clustering effect is better in high SNR conditions.

3.2. The ROC Curve under Constant False Alarm. In order to reflect the detection performance of the system model, we analyze the performance of the same clustering algorithm under different feature and the same feature under different clustering algorithm.

\subsubsection{Detection Performance of Different Features under the Same Clustering Algorithm}

(1) Detection Performance of Different Features under KMeans Clustering Algorithm. Figures 7 and 8 show the ROC curves of the four different signal features combined with the $K$-means clustering algorithm in SNR $=-10$ and SNR $=-12$ conditions, respectively. Tables 1 and 2 show the detection probabilities of each feature when the false alarm probability is constant under different SNR condition. From Table 1, we can find that the detection performance with RMET, MSE, and $\mathrm{MME}$ as the energy feature ratio is improved by $2 \%, 5 \%$, and $9 \%$, respectively, under $\mathrm{SNR}=-10$ and $P f=0.1$. At the same time, it can be found that the detection performance of MME as a feature is better than other features under the premise of using $\mathrm{K}$-means clustering algorithm. Compared to the use of signal energy as a feature, the feature of the random matrix can better distinguish the signal and the noise which can improve the classification result of the clustering algorithm and improve the performance of the spectrum sensing.

(2) Detection Performance of Different Characteristics under K-Medoids Clustering Algorithm. Figures 9 and 10 show the ROC curves of the four different features combined with the $K$-medoids clustering algorithm at $\mathrm{SNR}=-10$ and $\mathrm{SNR}=-12$, respectively. Tables 3 and 4 show the detection probabilities 


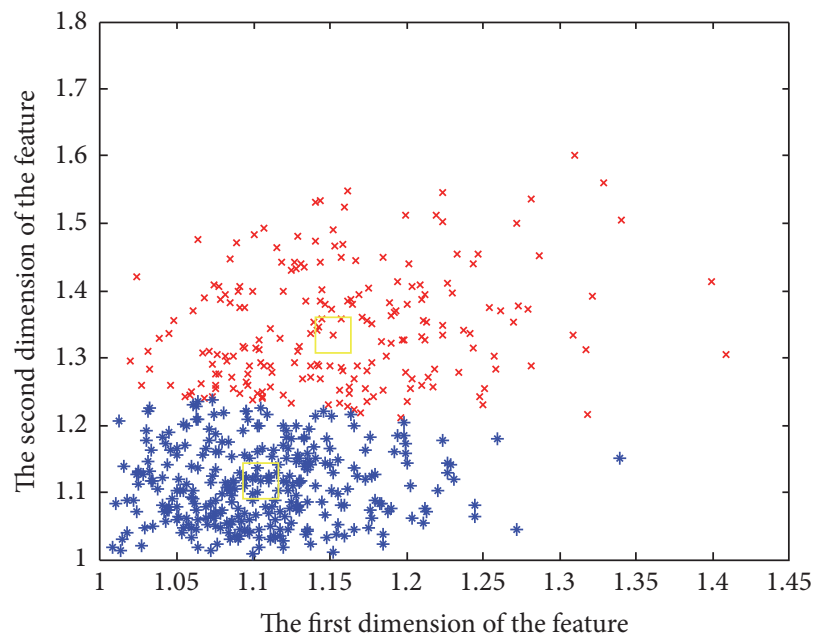

FIGURE 5: The clustering map under $K$-medoids algorithm.

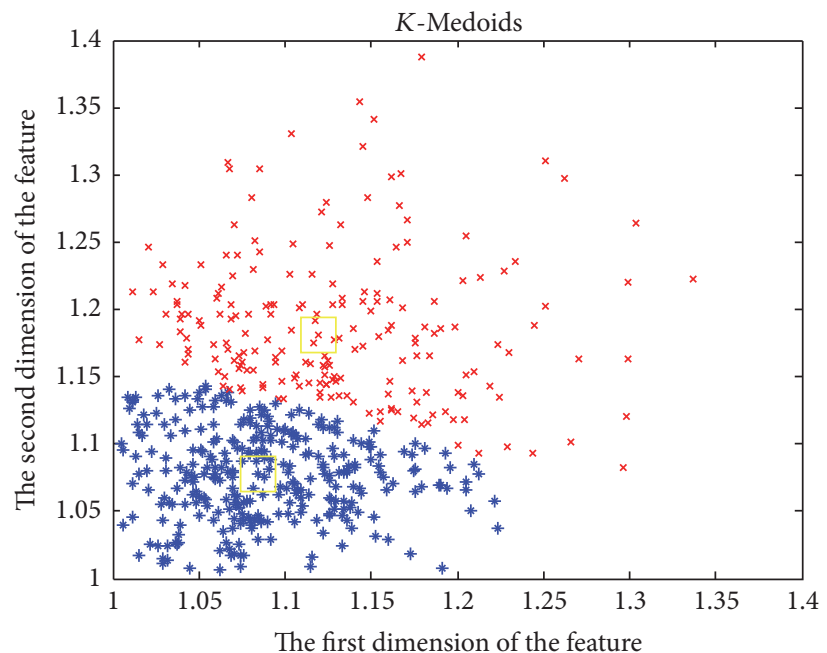

Figure 6: The clustering map under $K$-medoids algorithm.

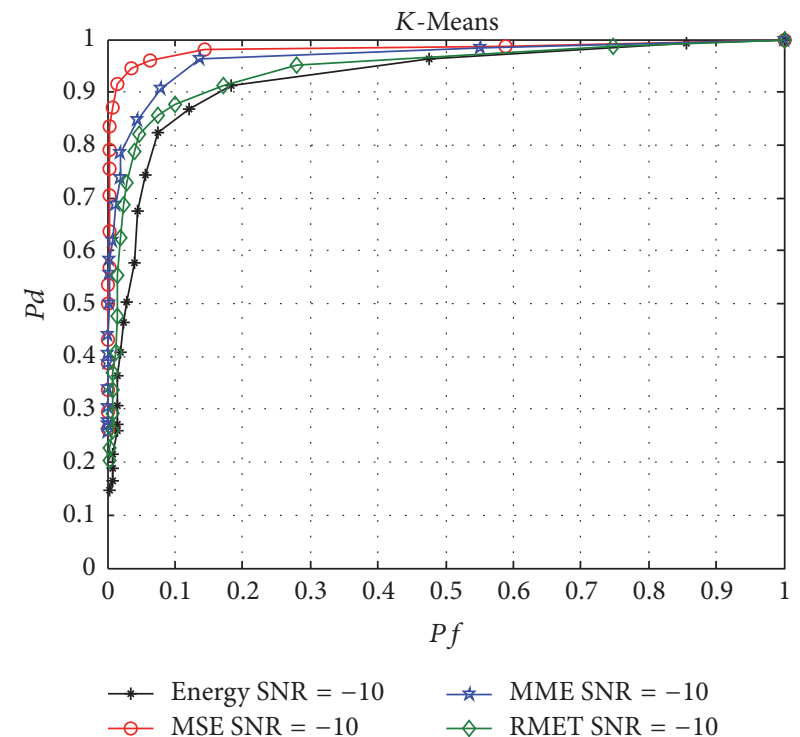

Figure 7: ROC curve of different features in SNR $=-10$. 


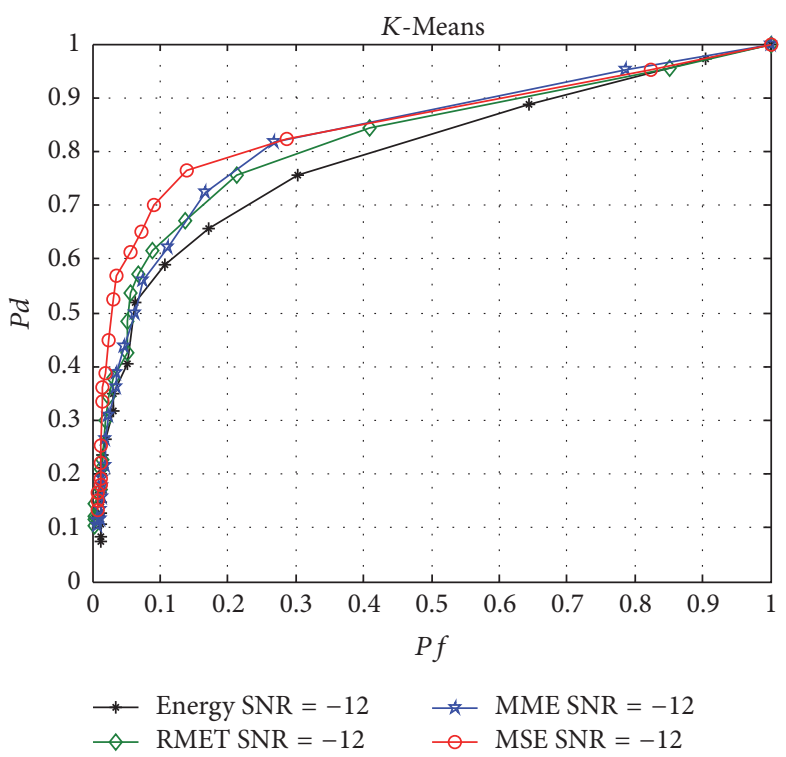

FIGURE 8: ROC curve of different features in $\mathrm{SNR}=-12$.

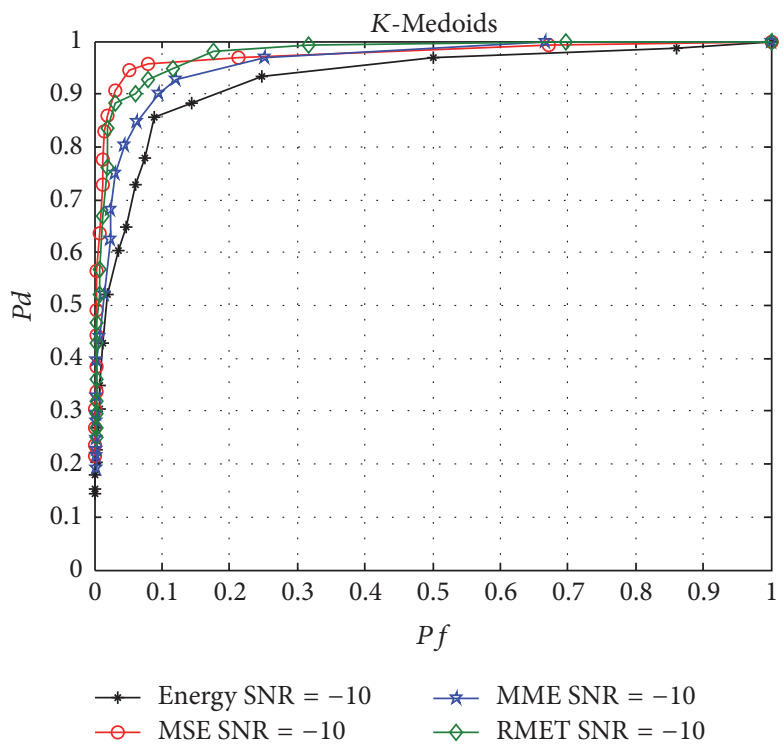

FIGURE 9: ROC curve of different features in $\mathrm{SNR}=-10$.

TABLE 1: The $P d$ comparison of different features in $S N R=-10$.

\begin{tabular}{lcc}
\hline Feature & $P f=0.1$ & $P f=0.2$ \\
\hline Energy & 0.85 & 0.91 \\
RMET & 0.87 & 0.92 \\
MME & 0.93 & 0.96 \\
MSE & 0.90 & 0.98 \\
\hline
\end{tabular}

of each feature when the false alarm probability is constant at different SNR conditions. From Table 3, we can calculate that the detection performance with RMET, MSE, and MME as the energy feature ratio is improved by $8 \%, 4 \%$, and $10 \%$, respectively, under SNR $=-10$ and $P f=0.1$. The detection
TABLE 2: The $P d$ comparison of different features in $\mathrm{SNR}=-12$.

\begin{tabular}{lcc}
\hline Feature & $P f=0.1$ & $P f=0.2$ \\
\hline Energy & 0.56 & 0.67 \\
RMET & 0.63 & 0.74 \\
MME & 0.60 & 0.75 \\
MSE & 0.71 & 0.78 \\
\hline
\end{tabular}

probability of using MME, MSE, and RMET as a feature is higher than energy feature. At the same time, it can be found that the detection performance of MSE as a feature is better than other features under the premise of using $K$ means clustering algorithm. 


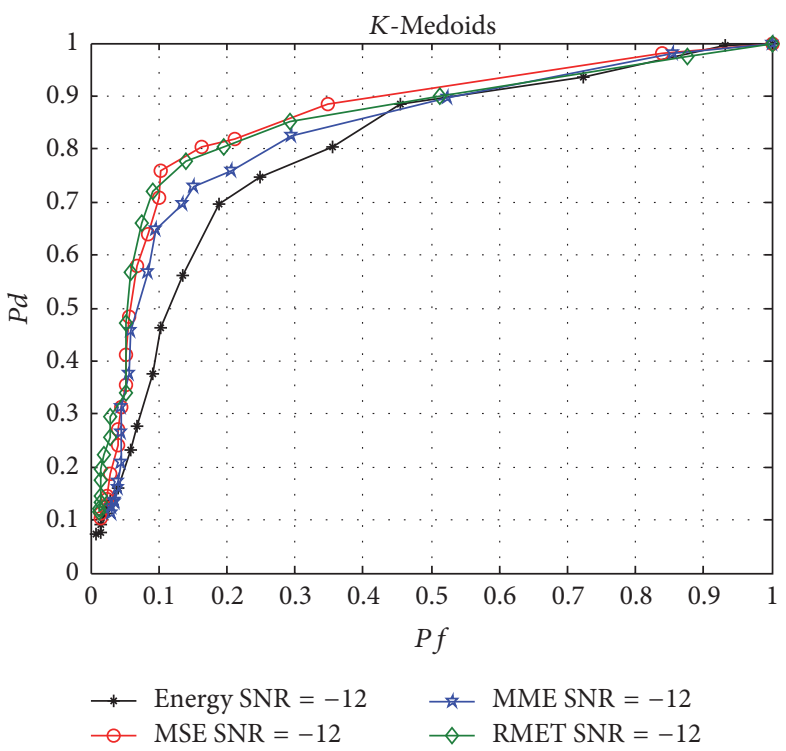

FIGURE 10: ROC curve of different features in SNR $=-12$.

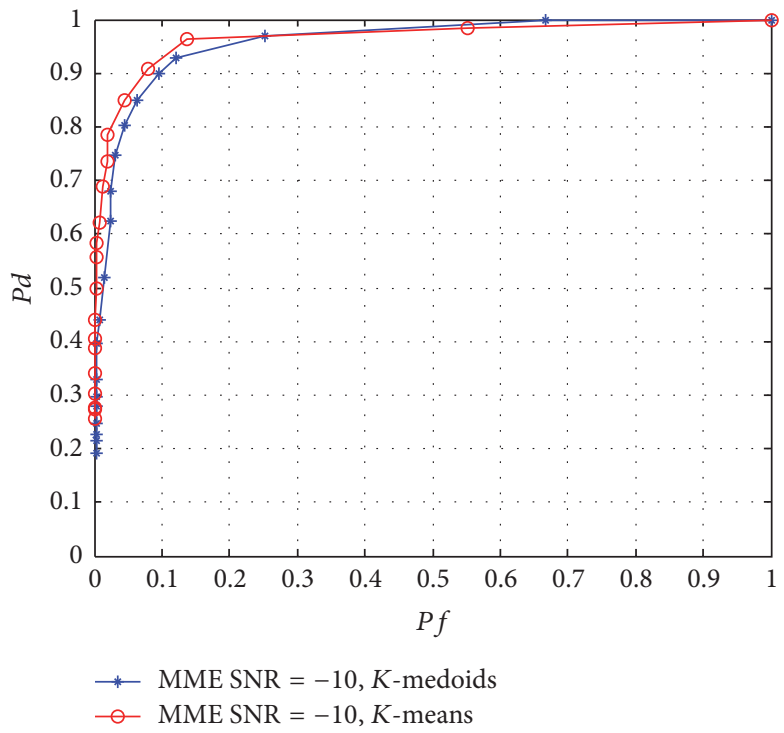

FIGURE 11: MME ROC curve of different algorithms in SNR $=-10$.

TABLE 3: The $P d$ comparison of different features in $\mathrm{SNR}=-10$

\begin{tabular}{lcc}
\hline Feature & $P f=0.1$ & $P f=0.2$ \\
\hline Energy & 0.86 & 0.91 \\
RMET & 0.93 & 0.98 \\
MME & 0.90 & 0.95 \\
MSE & 0.95 & 0.96 \\
\hline
\end{tabular}

\subsubsection{Comparison of Detection Performance of the Same} Feature under Different Clustering Algorithms

(1) Performance Comparison of MME Characteristics under Two Kinds of Clustering Algorithms. It can be seen from Figure 11 that, under SNR $=-10$ conditions, the sensing
TABLE 4: The $P d$ comparison of different features in SNR $=-12$.

\begin{tabular}{lcc}
\hline Feature & $P f=0.1$ & $P f=0.2$ \\
\hline Energy & 0.45 & 0.71 \\
RMET & 0.73 & 0.81 \\
MME & 0.65 & 0.75 \\
MSE & 0.71 & 0.82 \\
\hline
\end{tabular}

performance of $K$-means clustering algorithm is better than $K$-medoids clustering algorithm.

(2) Performance Comparison of MSE Features under Two Kinds of Clustering Algorithms. It can be seen from Figure 12 that, under SNR $=-10$ conditions, the sensing performance 


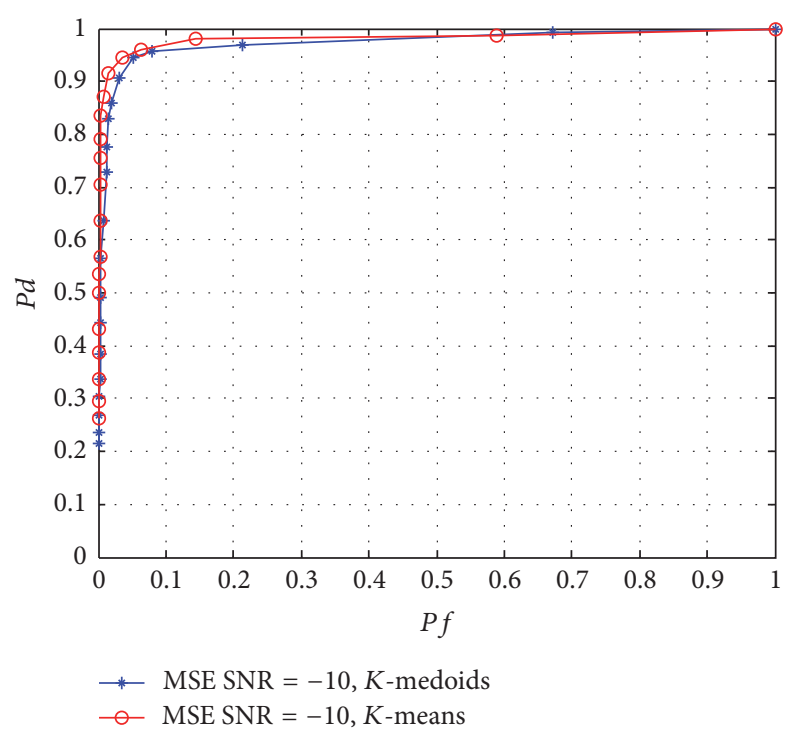

FIGURE 12: MSE ROC curve of different algorithms in SNR $=-10$.

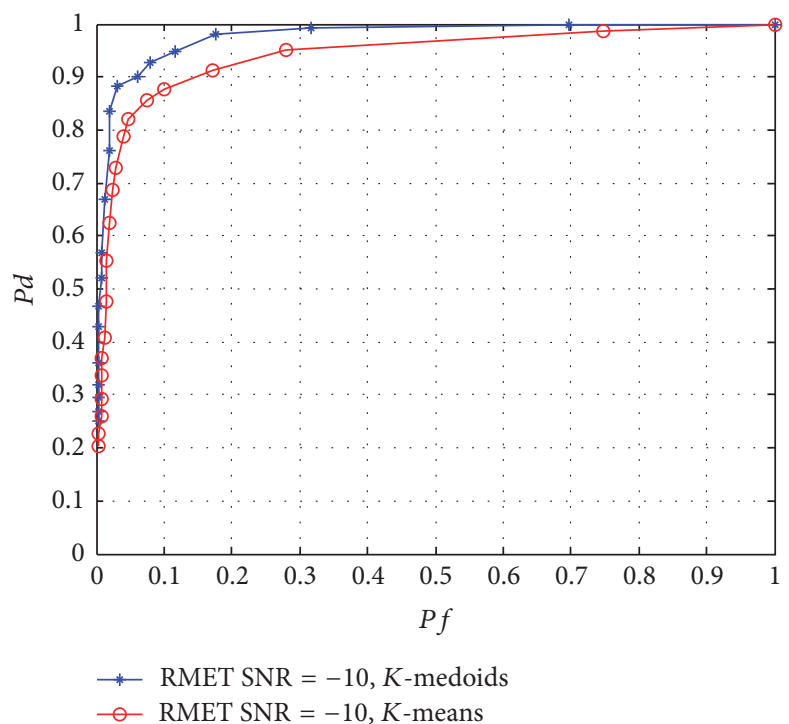

FIGURE 13: RMET ROC curve of different algorithms in SNR $=-10$.

of $K$-means clustering algorithm is better than $K$-medoids clustering algorithm.

(3) Performance Comparison of RMET Features under Two Kinds of Clustering Algorithms. It can be seen from Figure 13 that, under SNR $=-10$ conditions, the sensing performance of $K$-medoids clustering algorithm is better than $K$-means clustering algorithm.

From Figures 11-13, we can find that when MSE and MME features are used, the $K$-means clustering algorithm will have better detection performance and when RMET feature is used, the $K$-medoids clustering algorithm will have better performance.
3.2.3. Summary. According to the analysis of Section 3.2, we can make the following conclusions on how to choose eigenvalues and clustering algorithms.

Regardless of the clustering algorithm, RMET, MME, or MSE is better than using signal energy as a feature.

When using the $k$-means algorithm, select the MSE feature, which will get the best sensing results.

If the $k$-medoids algorithm is used as the clustering algorithm, selecting RMET as the feature can obtain the best sensing results.

3.3. ROC Curves under Different Collaboration Users. Figures 14 and 15 show the detection performance of the system under 


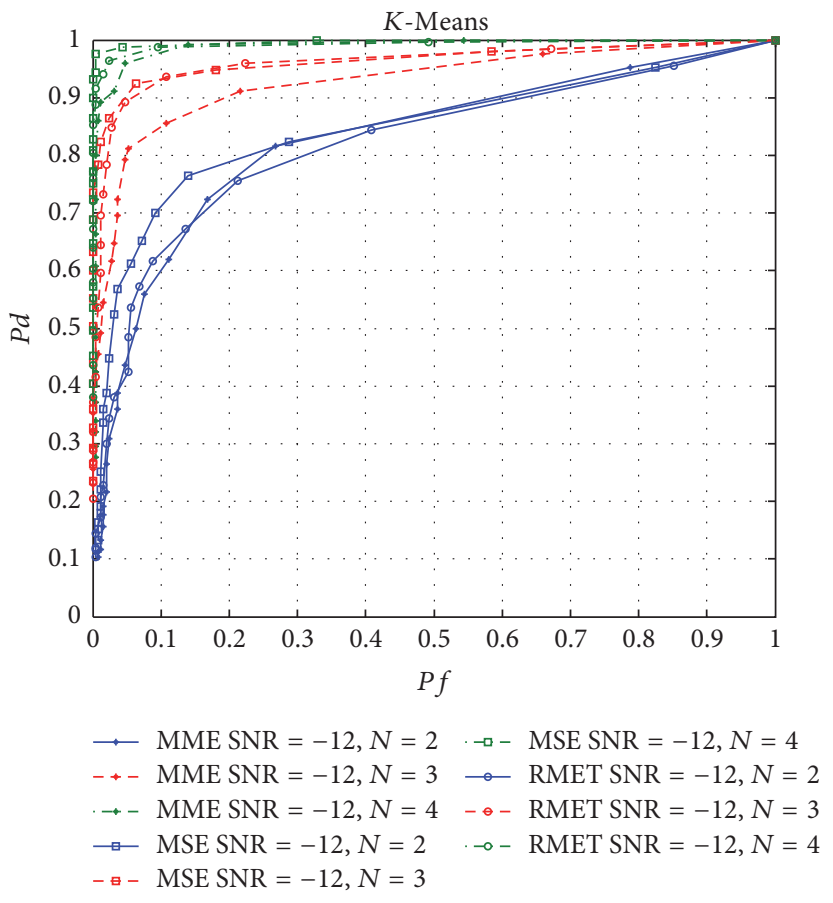

FIGURE 14: The ROC curves of different collaboration users.

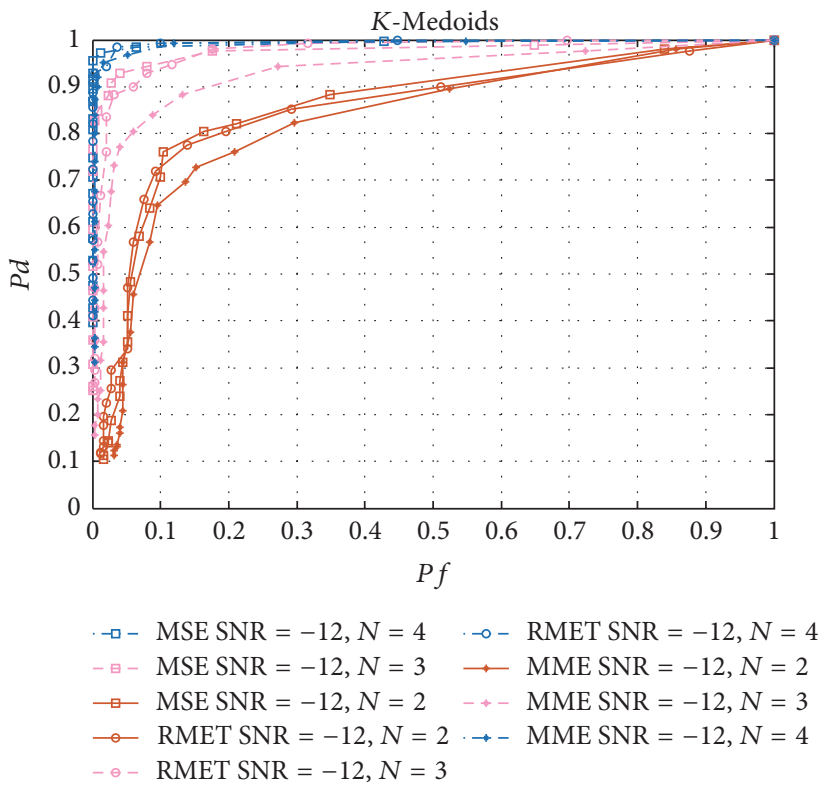

Figure 15: The ROC curves of different collaboration users.

different number of collaboration users. We can find that, regardless of the use of that feature or clustering algorithm, with the increase in the number of collaboration users, the sensing performance of the system will be better.

\section{Conclusions}

In this paper, we propose a machine learning spectrum sensing method based on signal features (Algorithm 1). The main content is using clustering algorithm to classify the signal features and then in the experimental part we analyzed the sensing performance of the three different features under the same clustering algorithm. The experimental results show that these three signal features have better effects than using signal energy as a feature. We also analyzed the detection performance of the same feature under different clustering algorithms. The experimental show that each feature has different performance in different conditions. In practice, we should select the suitable signal feature and clustering algorithm according to the current conditions. 
Input: (1) Train data $\left(T_{1}, T_{2}, \ldots, T_{N}\right)$ and test data $\left(\widehat{T}_{1}, \widehat{T}_{2}, \ldots, \widehat{T}_{n}\right)$

(2) Calculate the distanced between $T_{i}$ and $\varphi$, get the minimum value of $d$ and reclassify it.

(3) Calculate the mean of all points in the cluster and use the mean as the centroid Or select the smallest points of distance from one to all points as centroids.

(4) Calculate $J=\sum_{k=1}^{K} \sum_{z_{j} \in \varphi_{k}}\left\|Z_{j}-\varphi_{k}\right\|^{2}$

(5) If the $J$ converges then algorithm will stops, otherwise it return the second step.

(6) Import the test data $\left(\widehat{T}_{1}, \widehat{T}_{2}, \ldots, \widehat{T}_{n}\right)$

(7) If $\left\|\widehat{T}-\varphi_{1}\right\| / \min _{K=2,3, \ldots, k, k}\left\|\widehat{T}-\varphi_{k}\right\| \geq \varepsilon$ then output $A=1$, otherwise output $A=0$.

Algorithm 1: Spectrum sensing method based on signal feature and clustering algorithm.

\section{Conflicts of Interest}

The authors declare that they have no conflicts of interest.

\section{Acknowledgments}

This work was supported in part by the Degree and Graduate Education Reform Project of Guangdong Province (Grant no. 2016JGXM_MS_26), the Foundation of Key Laboratory of Machine Intelligence and Advanced Computing of the Ministry of Education (Grant no. MSC-201706A), the Science and Technology Program of Guangdong Province (Grant no. 2016B090918031), the Central Government's Special Funds in Support of the Development of Local Colleges and Universities, and Higher Education Quality Project of Guangdong Province.

\section{References}

[1] Z. Quan, S. Cui, and A. H. Sayed, "Optimal linear cooperation for spectrum sensing in cognitive radio networks," IEEE Journal of Selected Topics in Signal Processing, vol. 2, no. 1, pp. 28-40, 2008.

[2] Y. Zeng and Y.-C. Liang, "Eigenvalue-based spectrum sensing algorithms for cognitive radio," IEEE Transactions on Communications, vol. 57, no. 6, pp. 1784-1793, 2009.

[3] L. S. Cardoso, M. Debbah, P. Bianchi, and J. Najim, "Cooperative spectrum sensing using random matrix theory," in Proceedings of the 3rd International Symposium on Wireless Pervasive Computing, (ISWPC '08), pp. 334-338, May 2008.

[4] A. Taherpour, M. Nasiri-Kenari, and S. Gazor, "Multiple antenna spectrum sensing in cognitive radios," IEEE Transactions on Wireless Communications, vol. 9, no. 2, pp. 814-823, 2010.

[5] L. S. Cardoso, M. Debbah, P. Bianchi, and J. Najim, "Cooperative spectrum sensing using random matrix theory," in Proceedings of the 3rd International Symposium on Wireless Pervasive Computing, ISWPC 2008, pp. 334-338, May 2008.

[6] V. Marčenko A and A. Pastur L, "Distribution of eigenvalues for some sets of random matrices," Mathematics of the USSRSbornik, vol. 1, no. 4, p. 457, 1967.

[7] V. Kumar, D. C. Kandpal, M. Jain, R. Gangopadhyay, and $S$. Debnath, " $K$-mean clustering based cooperative spectrum sensing in generalized $k$-Fading channels," in Proceedings of the 22nd National Conference on Communication, (NCC '16), March 2016.
[8] O. N. Feldheim and S. Sodin, "A universality result for the smallest eigenvalues of certain sample covariance matrices," Geometric and Functional Analysis, vol. 20, no. 1, pp. 88-123, 2010.

[9] H. Xue and F. Gao, "A machine learning based spectrumsensing algorithm using sample covariance matrix," in Proceedings of the 10th International Conference on Communications and Networking in China, CHINACOM 2015, pp. 476-480, August 2015.

[10] I. F. Akyildiz, B. F. Lo, and R. Balakrishnan, "Cooperative spectrum sensing in cognitive radio networks: a survey," Physical Communication, vol. 4, no. 1, pp. 40-62, 2011.

[11] Y. Song and Y. Zhou, "An improved spectrum sensing algorithm based on random matrix theory," in Proceedings of the 2017 19th International Conference on Advanced Communication Technology (ICACT), pp. 715-720, Feburary 2017. 


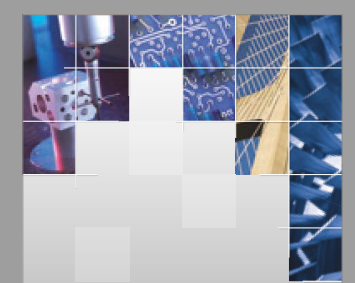

\section{Enfincering}
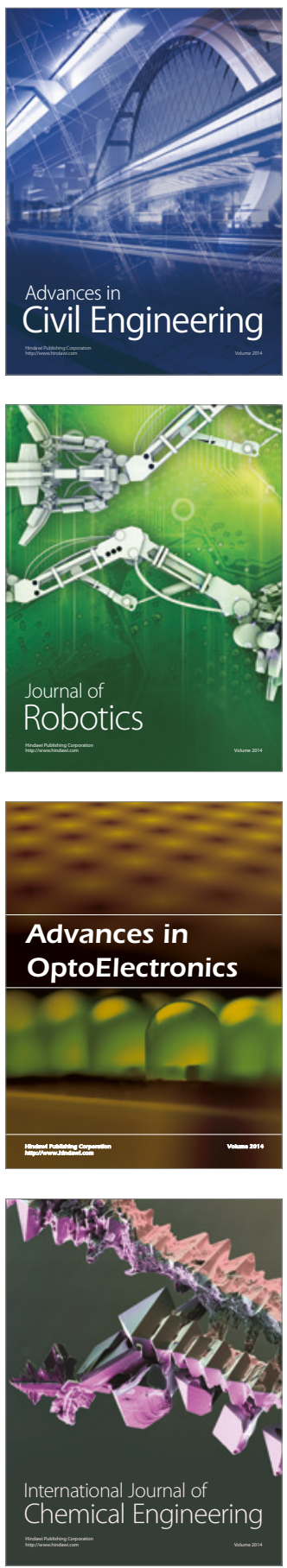

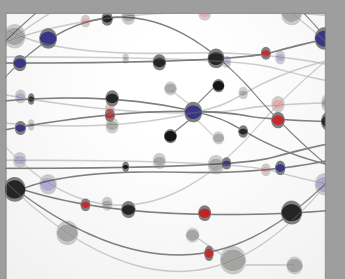

The Scientific World Journal

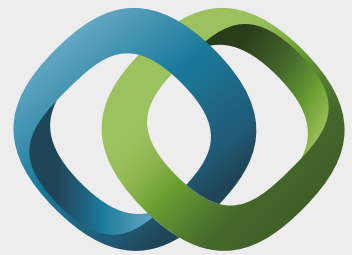

\section{Hindawi}

Submit your manuscripts at

https://www.hindawi.com
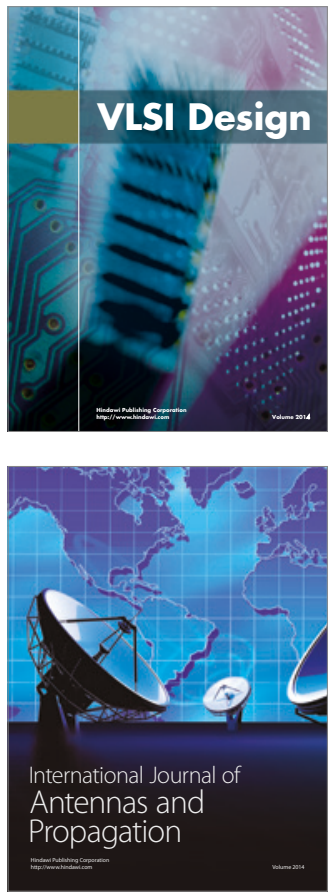

\section{Rotating}

Machinery
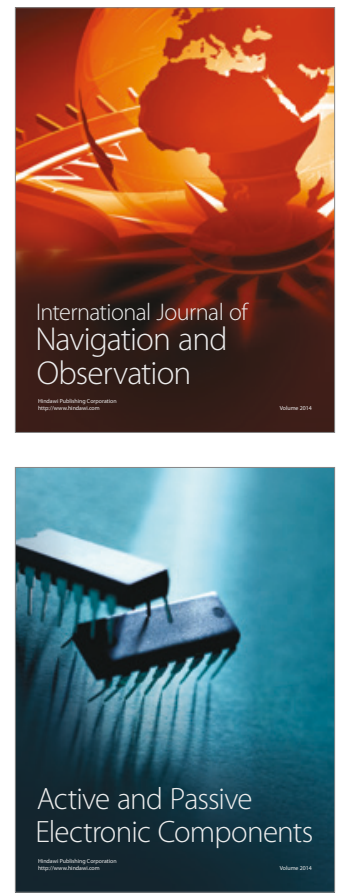
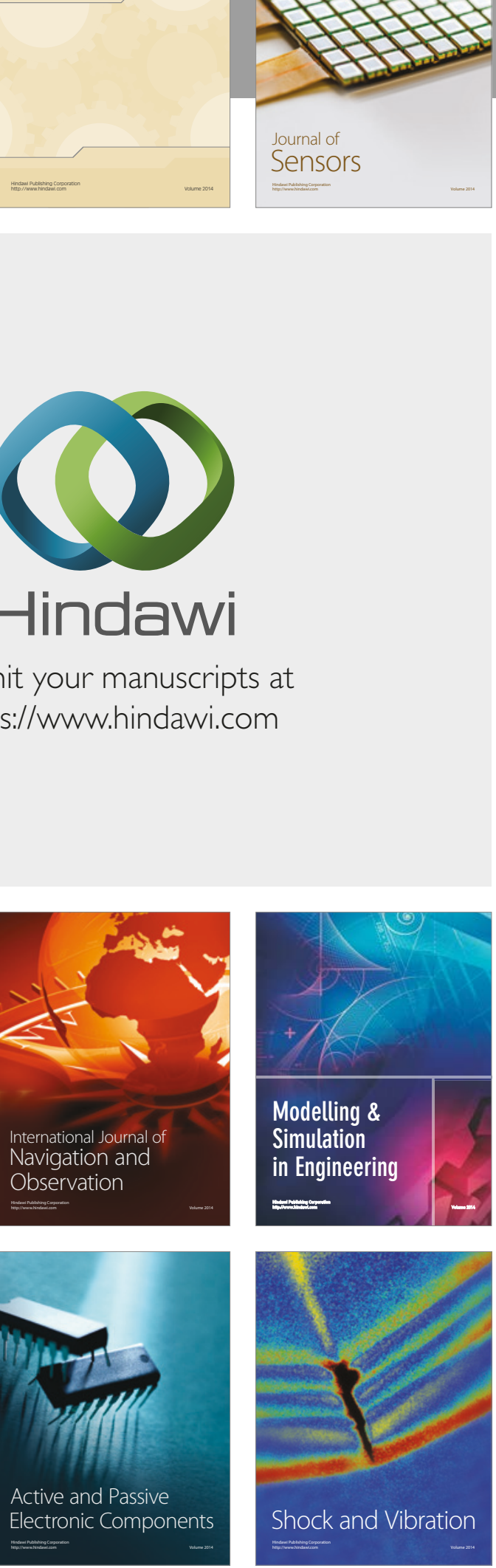
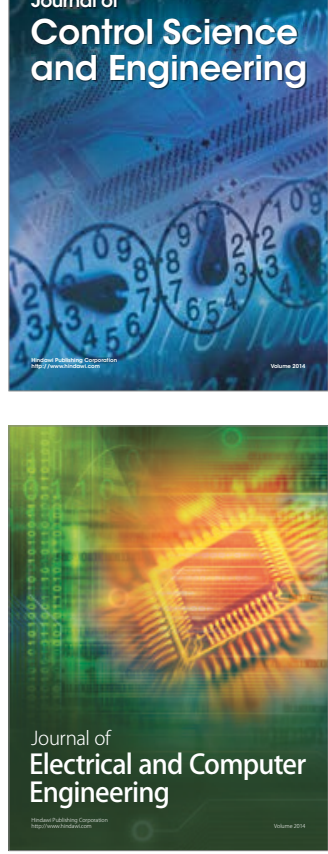

Distributed

Journal of

Control Science

and Engineering
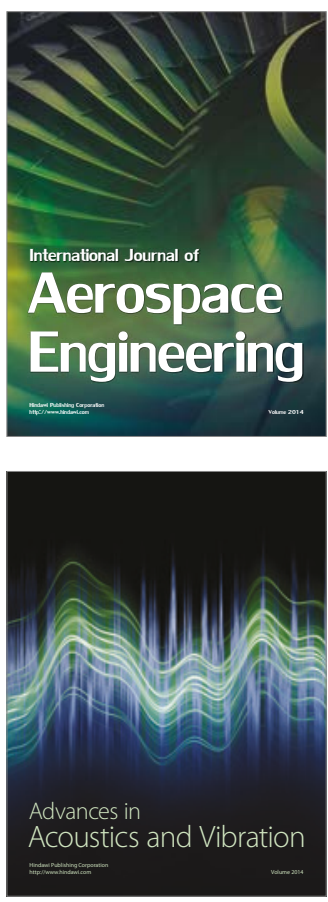

Sensor Networks 